\title{
Modèles explicatif et marginal de la stratégie de martelage d'une parcelle irrégulière
}

\author{
Max BRUCIAMACCHIE, Jean-Claude PIERRAT*, Julien TOMASINI \\ Laboratoire d'étude de la ressource Forêt-Bois, UMR INRA-ENGREF 1092, 14 rue Girardet, 54042 Nancy, France
}

(Reçu le 1er juin 2004 ; accepté le 24 janvier 2005)

\begin{abstract}
Résumé - Nous décrivons le comportement d'un marteleur pratiquant une sylviculture d'arbre dans une parcelle irrégulière. Nous étudions la séquence de décisions binaires (couper ou conserver un arbre) enregistrées dans l'ordre où elles sont arrêtées. Deux modèles répondant à des préoccupations différentes sont présentés. Avec le premier, ou modèle conditionnel, la décision prise sur un arbre est expliquée par les caractéristiques de l'arbre et des arbres dans son voisinage ainsi que par les décisions précédentes. La récolte globale et sa variabilité est estimée par simulation au niveau de la parcelle. Le second, ou modèle marginal, s'intéresse à la récolte globale et quantifie l'effet moyen des caractéristiques de l'arbre sur la probabilité de coupe. Il est couplé à un modèle de transition décrivant la dépendance aux décisions prises précédemment, au moyen de paramètres indépendants. Il permet de s'affranchir de «l'histoire » et du chemin choisi. Nous avons ainsi simulé un martelage correspondant à une récolte déterminée dans différentes hypothèses de dépendance entre décisions. Les paramètres de ces deux modèles ont été estimés avec les données du martelage d'un opérateur et différentes hypothèses de comportement ont été testées. Par simulation, nous étudions ensuite la variabilité des résultats du martelage selon les objectifs, selon le parcours emprunté et selon le paramètre de dépendance entre décisions.
\end{abstract}

sylviculture proche de la nature / traitement irrégulier / martelage / données binaires corrélées / modèle conditionnel / modèle marginal / modèle de transition marginalisé

\begin{abstract}
Explicative and marginal models for a marking strategy of a unevenaged stand. We describe the forester behavior selecting trees for thinning in a unevenaged stand. We have studied the binary decisions (cut or keep the tree) sequence, recorded in the order the decisions are made. The two models presented correspond to two different situations. First, in the conditional model, the decision concerning a tree is made according to its own properties and the properties of the trees next to it, as well as the decisions made before. The total cut and its variability are estimated by simulations on the scale of the stand. Secondly, the marginal model quantifies the averaged effect of the tree properties on the probability of cut. It is associated with a transition model describing the influence of the previous decisions and having independent parameters. It allows to break off the 'history' and the path followed. By simulation, we have marked trees corresponding to a determined cut, in different cases of decisions correlations. The parameters of the models have been estimated using forester data and different behavior hypothesis have been tested. By simulations, we have studied the variability of the cut according to its aims, depending on the path followed and the parameter relative to the decision correlations.
\end{abstract}

management based on natural process / marking / binary correlated data / conditional model / marginal model / marginalized transition model

\section{INTRODUCTION}

Le martelage est une opération importante en forêt irrégulière assurant à la fois la récolte, l'amélioration qualitative du peuplement, la régulation du couvert et des conditions locales de compétition [6,7]. Des dispositifs spécifiques («marteloscope ») ont été mis en place ces dernières années pour observer son déroulement, notamment lors de la formation des agents forestiers. Le traitement des données présente généralement l'évolution des principales variables dendrométriques selon différents scénarios sylvicoles $[1,2,7]$ mais peu d'études cherchent à décrire le comportement de l'opérateur «au pied de l'arbre ». Notre objectif ici a été de quantifier ce comportement et sa variabilité [8] en suivant un sylviculteur expert pratiquant une éclaircie.
Pour fixer les notations, appelons $y_{i}$ la décision du sylviculteur prise sur l'arbre $i$, avec $y_{i}=1$ lorsque l'arbre $i$ est coupé et $y_{i}=0$ dans le cas contraire ; notons $Y=\left(y_{1}, y_{2}, \ldots y_{I}\right)$ la séquence des décisions prises au fur et à mesure du cheminement dans la parcelle ; et notons $X$ le tableau dont les lignes $X_{i}=\left(x_{i 1}\right.$, $\left.x_{i 2}, \ldots x_{I K}\right)$ contiennent les valeurs de $K$ covariables mesurées sur l'arbre $i$. Nous nous proposons d'étudier $Y$ à la fois globalement et de façon progressive au cours du parcours.

En premier lieu, nous avons cherché à expliquer la décision $y_{i}$ prise sur l'arbre $i$ avec un modèle local impliquant les caractéristiques de l'arbre et des arbres de son voisinage ainsi que les décisions précédentes. Nous proposerons un modèle de la probabilité de coupe conditionnellement à ces facteurs. Assez classiquement, nous avons choisi un modèle dans lequel la

* Auteur pour correspondance : pierrat@ nancy-engref.inra.fr 
décision prise sur l'arbre $i$ dépend de $p$ décisions précédentes, $p$ étant un paramètre à déterminer.

En second lieu, nous intéressant directement à la récolte globale obtenue sur la parcelle, nous considérerons un modèle « marginal » de la probabilité de coupe. Cette probabilité, fonction uniquement des caractéristiques de l'arbre, pourra être interprétée comme étant l'un des objectifs du marteleur au niveau de la parcelle (par exemple, couper $20 \%$ des gros bois). D' autre part, pour l'inférence sur les paramètres, nous ferons intervenir les dépendances entre décisions dans un modèle de transition séparé. Ainsi que le propose Heagerty [3, 4], nous avons choisi une modélisation par chaîne de Markov dans lequel la décision prise sur l'arbre $i$ dépend des $p$ décisions précédentes.

Cet article sera organisé comme suit. Dans la section matériel et méthodes, nous présenterons l'origine des données, les modèles conditionnel et marginal, l'estimation de leurs paramètres. Nous présenterons ensuite l'utilisation de ces modèles pour analyser la variabilité des probabilités de coupe et de la récolte.

Dans la section résultats, ces méthodes seront tout d'abord appliquées aux données de martelage du sylviculteur ayant opéré. Nous donnerons les estimations et les intervalles de confiance des paramètres du modèle et testerons différents modèles.

Ensuite, à partir de simulations, nous étudierons numériquement la variabilité de la probabilité de coupe des individus, selon les décisions précédentes, selon le parcours et selon l'intensité de la dépendance entre décisions. Enfin, nous étudierons la variabilité de la récolte pour un comportement de l'opérateur donné ou pour un martelage correspondant à des objectifs déterminés.

En conclusion quelques perspectives seront évoquées.

\section{MATÉRIELS ET MÉTHODES}

\subsection{Présentation du marteloscope}

Le marteloscope de Flavigny, situé dans une forêt de plaine à base de feuillus, occupait originellement une surface de 3 ha, mais, suite aux chablis de 1999, nous n'avons conservé que la partie non endommagée de 2 ha contenant 400 arbres.

Pour chaque arbre, nous disposons des coordonnées, de l'essence (chêne, charme, érable, frêne, feuillus divers), du diamètre, de la qualité (classe A, B, C ou D selon les critères externes [1]) et du taux de rentabilité (accroissement de la valeur d'un arbre pour l'année suivante divisé par la valeur de l'arbre). Suite à une absence de gestion depuis plusieurs années, le matériel sur pied est élevé. Peu de tiges ont un potentiel d'avenir et environ $20 \%$ du volume est susceptible d'être prélevé, en priorité des arbres de faible qualité ou mûrs. Afin d'activer le renouvellement du peuplement, les perches peuvent être favorisées.

Un opérateur a pratiqué fictivement une coupe d'éclaircie de la parcelle en notant sur un plan son cheminement ainsi que les arbres désignés au fur et à mesure de son parcours.

\subsection{Modèles d'analyse du martelage}

\subsubsection{Modèle local (ou « conditionnel »)}

Un modèle de régression logistique a été utilisé pour modéliser la probabilité de coupe, en fonction des propres caractéristiques de l'arbre (l'essence, le taux de rentabilité, la qualité et le diamètre) et des caractéristiques des $p$ arbres précédents les plus proches géographiquement. Nous avons également fait intervenir la somme des diamètres coupés sur ces $p$ arbres (soit la somme des diamètres pondérés par les décisions prises).

Pour l'arbre $i$, nous noterons :

- les décisions prises sur les $p$ arbres les plus proches déjà visités $Y_{i}^{-}=\left(Y_{i(1)}, \ldots, Y_{i(p)}\right)$

- les caractéristiques des mêmes arbres $X_{i}^{-}=\left(X_{i(1)}, \ldots, X_{i(p)}\right)$

- $\mathrm{C}_{\mathrm{i}}$ la probabilité conditionnelle $\mathrm{E}\left(Y_{i} /\left(X_{i}, X_{i}^{-}, Y_{i}^{-}\right)\right)$. Le modèle s'écrit :

$$
\operatorname{logit}\left(C_{i}\right)=X_{i} a+X_{i}^{-} b+c \sum \operatorname{diam}_{i-j} \mathrm{Y}_{i-j} .
$$

Les vecteurs de paramètres $a, b$ et $c$ peuvent s'interpréter directement comme la variation sur l'échelle logit de la probabilité de coupe, lorsque les covariables varient d'une unité.

Pour le calcul de la vraisemblance des observations, nous ferons l'hypothèse que la dépendance au passé est contenue dans $X_{i}^{-}$et $Y_{i}^{-}$: $\mathrm{E}\left(Y_{i} / X\right.$ et $\left.(Y j, j<i)\right)=\mathrm{E}\left(Y_{i} /\left(X_{i}, X_{i}^{-}, Y_{i}^{-}\right)\right)$.

\subsubsection{Modèle marginal}

Nous avons choisi un modèle logistique pour décrire la probabilité de coupe d'un arbre possédant les covariables $X_{i}$. Un second modèle décrira la dépendance d'une décision $Y_{i}$ aux covariables $X_{i}$ et aux décisions précédentes [4]. Pour des raisons de calcul et à la différence du modèle (1), nous avons fait intervenir les décisions immédiatement précédentes dans le temps, sans contrainte géographique.

En notant $M_{i}$ la probabilité marginale $E\left(Y_{i} / X_{i}\right)$, le modèle marginal s'écrira :

$$
\operatorname{logit}\left(M_{i}\right)=X_{i} \alpha
$$

En notant $Z$ le vecteur des $p$ décisions précédentes et en notant $T_{i}$ la probabilité de coupe $E\left(Y_{i} / X_{i}\right.$ et $\left.Z\right)$ le modèle de transition s'écrira :

$$
\operatorname{logit}\left(T_{i}\right)=d_{i}+g \sum \operatorname{diam}_{i-j} Z_{i-j}
$$

Ici encore, l'influence d'une décision est pondérée par le diamètre de l'arbre sur lequel elle est prise.

Quelques remarques peuvent être faites :

- Pour le calcul de la vraisemblance des observations, nous ferons l'hypothèse markovienne (la dépendance aux décisions passées est résumée dans la dépendance aux $p$ décisions précédentes).

- $d_{i}$ n'est pas un paramètre disponible. Une fois connus les paramètres $\alpha$ et $g$, la valeur de $d_{i}$ est déterminée pour que $M_{i}$ soit la moyenne de $T_{i}$ sur la distribution de $Z$. Ce calcul est détaillé en annexe.

- Le modèle marginal (2) décrit l'influence moyenne des caractéristiques $X_{i}$ sur le logit $\left(M_{i}\right)$ et résume des objectifs globaux.

Le paramètre $g$ précise l'influence moyenne des décisions précédentes et détermine la fluctuation de la probabilité de coupe autour de la valeur moyenne donnée par (2). Néanmoins, ce modèle ne s'applique pas à un arbre. Son intérêt est d'évaluer l'effet des covariables en s'affranchissant de «l'histoire » et donc en particulier du chemin choisi par le sylviculteur.

\subsection{Estimation des paramètres}

Les paramètres ont été estimés par la méthode du maximum de vraisemblance, ce qui permet de disposer des tests statistiques classiques de comparaison de modèles, en particulier de ceux basés sur le rapport de vraisemblance. D'autre part, du fait des liens logit, les paramètres peuvent prendre des valeurs quelconques, ce qui simplifie la procédure d'estimation. 
Pour le modèle (1), il est possible d'utiliser un logiciel de régression standard tel que Splus. Pour les modèles (2) et (3), nous avons maximisé numériquement la vraisemblance en utilisant la méthode du simplexe [5]. Cet algorithme demande en entrée des valeurs initiales pour les paramètres et une routine effectuant le calcul de la vraisemblance $\mathrm{V}$ pour les valeurs des paramètres fixées (les dérivées ne sont pas nécessaires avec cet algorithme). Cette routine est présentée en annexe.

\section{4. Étude de la variabilité des probabilités locales}

Avec le modèle (1), une analyse arbre par arbre de la probabilité de coupe peut être effectuée.

\subsubsection{Selon les décisions précédentes}

Lorsque les paramètres $a, b, c$ sont connus, la probabilité de coupe de l'arbre $i$, possède une valeur moyenne sur toutes les décisions précédentes possibles $P_{i}=E\left(Y_{i} /\left(X_{i}, X_{i}^{-}\right)\right)$et une variabilité qui dépend de la loi de probabilité des décisions précédentes.

Nous avons calculé les $P_{i}$ par simulation. Pour cela, nous avons répété la simulation de la séquence $Y$ des décisions prises selon un parcours et obtenu $P_{i}$ par la moyenne des décisions ( 0 ou 1$)$ prises sur l'arbre $i$. Pour atteindre la stabilité, nous avons répété le processus 10000 fois.

Une séquence de décisions $Y$ complète a été simulé en considérant chaque arbre successivement dans l'ordre du parcours : les $p$ premiers arbres sont conservés ; pour chaque arbre suivant, la probabilité $C i$ est calculée par (1) puis la décision $y_{i}$ est simulée en décidant la coupe avec la probabilité $C i$.

Les différences entre $\mathrm{Ci}-P_{i}$ permettent de repérer les arbres dont la probabilité de coupe a été la plus influencée par les décisions prises sur les arbres voisins lors du passage particulier de l'opérateur (pour lequel $C i$ est obtenu).

L'indicateur $\operatorname{Var}(i)=\Sigma\left(C i-P_{i}\right)^{2} \times P\left(Y_{i}^{-}=q\right)$, soit la variance des probabilités conditionnelles de coupe de l'arbre $i$, permet de préciser les fluctuations possibles pour l'arbre i et donc de déterminer les arbres les plus susceptibles d'être influencés par leur voisinage.

\subsubsection{Selon le parcours}

Considérant le même opérateur (paramètres identiques) empruntant un chemin différent, nous présenterons $\operatorname{Var}(i)$ pour chaque arbre. Notons que le changement de voisins fait à la fois changer $X_{i}^{-}$et la loi de probabilité de $Y_{i}^{-}$.

\subsubsection{Selon l'intensité de la dépendance entre décisions (paramètre c)}

Intuitivement, lorsque le paramètre $c$ augmente une plus grande dispersion est attendue : nous présenterons $\operatorname{Var}(i)$ en fonction du paramètre $c$.

\section{5. Étude de la récolte par simulation de martelage}

\subsubsection{Simulation de comportements de l'opérateur}

Pour donner une estimation de la récolte et de sa variabilité, le modèle de comportement (1) a été simulé en considérant chaque arbre pris successivement le long du parcours. Différentes valeurs des paramètres voisines de celles de l'opérateur ont été envisagées. À titre d'exemple, nous présenterons les effectifs coupés par qualité lorsque le paramètre c varie.

\subsubsection{Simulation à objectifs fixés}

Le modèle marginal permet de simuler directement un martelage correspondant à des objectifs d'éclaircie fixés. Nous prendrons un exemple où des effectifs par catégorie diamètre × qualité (Tab. III) sont recherchés pour une certaine vente.

Les paramètres $\alpha$ du modèle marginal sont déterminés en mettant les proportions de coupe sous la forme logit (Tab. III). Les valeurs des paramètres $g$ et le parcours de la parcelle sont à la disposition de l'opérateur : une propriété du modèle est que pour l'arbre $i$, la valeur moyenne est égale à la marge $\mathrm{Mi}$, pour tous les parcours empruntés et pour toutes valeurs de $g$. Nous pouvons donc faire varier le paramètre de dépendance entre décisions sans faire varier l'objectif de récolte.

Nous présenterons les résultats, en nous intéressant surtout à leurs écarts-types.

Une simulation a été conduite de la manière suivante.

Initialement, les $p$ premiers arbres sont conservés.

Pour chaque arbre suivant, les valeurs de $d_{i}$, puis celles de $T_{i}$ sont déterminées. La coupe de l'arbre est alors déterminée avec la probabilité $T_{i}$. Après avoir simulé 1000 fois la séquence de décisions $Y=$ $\left(y_{3}, y_{4}, \ldots, y_{I}\right)$, nous avons calculé la moyenne des 1000 décisions $(0$ ou 1) pour chaque arbre pour obtenir la coupe moyenne.

\section{RÉSULTATS}

\subsection{Analyse du martelage d'un sylviculteur}

\subsubsection{Analyse du comportement par le modèle conditionnel}

Nous avons tout d'abord choisi $p$, le nombre de voisins intervenant dans l'explication de la probabilité de coupe. Le tableau Ia présente les différents modèles pour $p$ variant de 0 jusqu'à 4 ainsi que le modèle qui les contient tous.

Sur le critère de la vraisemblance, le meilleur modèle est celui faisant intervenir les décisions prises sur les 3 plus proches voisins. Par rapport au modèle d' ordre 0 , le coefficient est significatif avec une déviance de $\mathrm{D}=2 \times(121,0-118,8)=4,4$ soit une $p$ value de 5,5\%.

Une fois cette variable d' ordre 3 incluse, il est inutile de faire intervenir les autres ordre $\mathrm{D}=2 \times(118,8-118,38)=1$ avec 3 degrés de liberté.

Pour tous les ordres, le coefficient relatif aux décisions précédentes est négatif. Un arbre a donc une probabilité inférieure d'être prélevé lorsque des arbres proches ont été coupés ou autrement dit, lorsque l'opérateur a dispersé les prélèvements dans toute la parcelle.

On remarque également que les autres coefficients du modèle sont stables pour les différents ordres.

Après simplification, le modèle retenu est présenté tableau $\mathrm{Ib}$, avec les intervalles de confiance à $5 \%$ sur les paramètres. Les vraisemblances permettent notamment de tester les variables « qualité du plus proche voisin » et « décisions précédentes ». En effet, les rapports de vraisemblance valent respectivement :

$$
\begin{aligned}
& (D=2 \times(122,84-120,39)=4,9, p \text { value à } 1 \text { d.d. } 1=3,7 \%) \text { et } \\
& (D=2 \times(123,4-120,39)=6,02, p \text { value à } 1 \text { d.d. } 1=1,5 \%) .
\end{aligned}
$$


Tableau Ia. Estimation (intervalle de confiance) des paramètres du modèle conditionnel.

\begin{tabular}{|c|c|c|c|c|c|c|}
\hline & Grand modèle & $\begin{array}{l}\text { Modèle } \\
\text { ordre } 4\end{array}$ & $\begin{array}{l}\text { Modèle } \\
\text { ordre } 3\end{array}$ & $\begin{array}{l}\text { Modèle } \\
\text { ordre } 2\end{array}$ & $\begin{array}{l}\text { Modèle } \\
\text { ordre } 1\end{array}$ & $\begin{array}{l}\text { Modèle } \\
\text { ordre } 0\end{array}$ \\
\hline Constante & $-5,85$ & $-6,33$ & $-6,0$ & $-5,70$ & $-5,28$ & $-6,36$ \\
\hline \multicolumn{7}{|c|}{ Caractéristiques propre à l'arbre } \\
\hline Taux & $\begin{array}{c}-23,6 \\
(-34,2 ;-15,0)\end{array}$ & $-23,6$ & $-24,0$ & $-24,1$ & $-24,6$ & $-24,3$ \\
\hline Diamètre $<20$ & 0 & 0 & 0 & 0 & 0 & 0 \\
\hline $20<$ diamètre $<40$ & $\begin{array}{c}2,64 \\
(2,1 ; 3,1)\end{array}$ & 2,87 & 2,9 & 2,51 & 2,32 & 2,71 \\
\hline $40<$ diamètre $<60$ & $\begin{array}{c}4,0 \\
(3,4 ; 4,5)\end{array}$ & 4,3 & 4,3 & 3,87 & 3,63 & 4,15 \\
\hline $60<$ diamètre & $\begin{array}{c}5,0 \\
(4,2 ; 5,8)\end{array}$ & 5,3 & 5,2 & 4,84 & 4,55 & 5,16 \\
\hline Qualité (A + B) & 0 & 0 & 0 & 0 & 0 & 0 \\
\hline Qualité C & $\begin{array}{c}2,1 \\
(1,6 ; 2,5)\end{array}$ & 2,1 & 2,1 & 2,0 & 1,7 & 2,12 \\
\hline Qualité D & $\begin{array}{c}2,5 \\
(2,0 ; 2,9)\end{array}$ & 2,5 & 2,4 & 2,41 & 2,1 & 2,52 \\
\hline Charme (0 ou 1$)$ & $\begin{array}{c}0,92 \\
(0,39 ; 1,4)\end{array}$ & 0,93 & 0,92 & 0,88 & 0,88 & 0,93 \\
\hline \multicolumn{7}{|c|}{ Caractéristiques des arbres précédents les plus proches } \\
\hline $\begin{array}{l}\text { Diamètre }<40 \\
(i-1)\end{array}$ & $\begin{array}{c}-0,07 \\
(-0,3 ; 0,47)\end{array}$ & 0,27 & 0,15 & 0,16 & 0,09 & 0,29 \\
\hline $\begin{array}{l}\text { Qualité C + D } \\
(i-1)\end{array}$ & $\begin{array}{c}-1,1 \\
(-1,5 ;-0,7)\end{array}$ & $-1,2$ & $-1,1$ & $-1,1$ & $-1,0$ & $-1,2$ \\
\hline $\begin{array}{l}\text { Diamètre }<40 \\
(i-2)\end{array}$ & $\begin{array}{c}0,00 \\
(-0,17 ; 0,2)\end{array}$ & 0,00 & $-0,02$ & 0,01 & 0,14 & 0,23 \\
\hline $\begin{array}{l}\text { Qualité C + D } \\
(i-2)\end{array}$ & $\begin{array}{c}0,06 \\
(-0,28 ; 0,40)\end{array}$ & 0,1 & 0,15 & 0,11 & $-0,06$ & 0,04 \\
\hline $\begin{array}{l}\text { Diamètre }<40 \\
(i-3)\end{array}$ & $\begin{array}{c}0,47 \\
(0,08 ; 0,84)\end{array}$ & 0,49 & 0,44 & 0,57 & 0,57 & 0,57 \\
\hline $\begin{array}{l}\text { Qualité C + D } \\
(i-3)\end{array}$ & $\begin{array}{c}-0,14 \\
(-0,50 ; 0,21)\end{array}$ & $-0,17$ & $-0,14$ & $-0,28$ & $-0,18$ & $-0,20$ \\
\hline $\begin{array}{l}\text { Diamètre }<40 \\
(i-4)\end{array}$ & $\begin{array}{c}0,53 \\
(0,12 ; 0,93)\end{array}$ & 0,35 & 0,41 & 0,36 & 0,36 & 0,41 \\
\hline $\begin{array}{l}\text { Qualité C + D } \\
(i-4)\end{array}$ & $\begin{array}{c}-0,40 \\
(-0,76 ;-0,05)\end{array}$ & $-0,17$ & $-0,24$ & $-0,12$ & $-0,18$ & $-0,19$ \\
\hline \multicolumn{7}{|c|}{ Cumul des diamètres coupés sur $p$ précédents les plus proches } \\
\hline Cumul sur $p=1$ arbre & $\begin{array}{c}-0,0049 \\
(-0,030 ; 0,01)\end{array}$ & & & & $-0,0163$ & \\
\hline Cumul sur $p=2$ arbres & $\begin{array}{c}0,0010 \\
(-0,013 ; 0,01)\end{array}$ & & & $-0,0134$ & & \\
\hline Cumul sur $p=3$ arbres & $\begin{array}{c}-0,0211 \\
(-0,033 ;-0,01)\end{array}$ & & $-0,0140$ & & & \\
\hline Cumul sur $p=4$ arbres & $\begin{array}{c}0,0087 \\
(-0,000 ; 0,01)\end{array}$ & $-0,0063$ & & & & \\
\hline Log vraisemblance & $-118,38$ & $-120,4$ & $-118,8$ & $-119,5$ & $-120,1$ & $-121,0$ \\
\hline
\end{tabular}

Globalement, les deux coefficients sont significatifs avec une déviance de $2 \times(126,0-120,39)=11,22, p$ value à 2 d.d.1. $=$ $0,5 \%$.

Quelques remarques peuvent être faites sur les autres coefficients.

Les prélèvements sont plus forts pour les arbres de faible taux de rentabilité, de gros diamètre et de faible qualité. Le charme est davantage coupé que les autres espèces. Les espèces, autres que le charme, ont mêmes coefficients (résultats non présentés). Les caractéristiques des arbres précédents ont peu d'influence, hormis la qualité de l'arbre le plus proche (qui a un effet négatif).

Les tentatives d'introduire la variable « surface terrière » du voisinage ou les distances entre arbres n'ont pas donné de résultats probants.

\subsubsection{Analyse marginale}

Le tableau II présente les estimations des coefficients du modèle marginal ainsi que leurs intervalles de confiance à $5 \%$ basés sur le rapport de vraisemblance. 
Tableau Ib. Estimation (intervalle de confiance) des paramètres du modèle conditionnel.

\begin{tabular}{|c|c|c|c|c|}
\hline & Modèle retenu & $\begin{array}{c}\text { Modele sans } \\
\text { caractéristiques } \\
\text { arbres précédents }\end{array}$ & $\begin{array}{l}\text { Modèle sans } \\
\text { décisions } \\
\text { précédentes }\end{array}$ & $\begin{array}{c}\text { Modèle } \\
\text { caractéristiques } \\
\text { de l'arbre }\end{array}$ \\
\hline Constante & $\begin{array}{c}-4,91 \\
(-5,8 ;-5,23)\end{array}$ & $\begin{array}{c}-6,21 \\
(-6,55 ;-5,92)\end{array}$ & $\begin{array}{c}-5,94 \\
(-6,3 ;-5,6)\end{array}$ & $\begin{array}{c}-6,57 \\
(-6,8 ;-6,2)\end{array}$ \\
\hline Taux & $\begin{array}{c}-24,1 \\
(-35,6 ;-16,0)\end{array}$ & $\begin{array}{c}-19,5 \\
(-31,4 ;-12,2)\end{array}$ & $\begin{array}{c}-20,6 \\
(-30,7 ;-11,6)\end{array}$ & $\begin{array}{c}-18,4 \\
(-28,4 ;-9,4)\end{array}$ \\
\hline $20<$ diamètre $<40$ & $\begin{array}{c}2,34 \\
(2,24 ; 3,0)\end{array}$ & $\begin{array}{c}2,64 \\
(2,1 ; 3,0)\end{array}$ & $\begin{array}{c}2,59 \\
(2,1 ; 3,0)\end{array}$ & $\begin{array}{c}2,50 \\
(2,0 ; 2,9)\end{array}$ \\
\hline $40<$ diamètre $<60$ & $\begin{array}{c}3,73 \\
(3,6 ; 4,7)\end{array}$ & $\begin{array}{c}3,9 \\
(3,3 ; 4,4)\end{array}$ & $\begin{array}{c}4,0 \\
(3,5 ; 4,6)\end{array}$ & $\begin{array}{c}3,84 \\
(3,3 ; 4,3)\end{array}$ \\
\hline $60<$ diamètre & $\begin{array}{c}4,57 \\
(4,2 ; 5,8)\end{array}$ & $\begin{array}{c}4,9 \\
(4,1 ; 5,6)\end{array}$ & $\begin{array}{c}5,1 \\
(4,3 ; 5,9)\end{array}$ & $\begin{array}{c}4,96 \\
(4,2 ; 5,7)\end{array}$ \\
\hline Qualité C & $\begin{array}{c}1,9 \\
(1,6 ; 2,6)\end{array}$ & $\begin{array}{c}1,94 \\
(1,5 ; 2,4)\end{array}$ & $\begin{array}{c}2,2 \\
(1,7 ; 2,7)\end{array}$ & $\begin{array}{c}2,1 \\
(1,6 ; 2,5)\end{array}$ \\
\hline Qualité D & $\begin{array}{c}2,32 \\
(2,0 ; 2,9)\end{array}$ & $\begin{array}{c}2,36 \\
(1,9 ; 2,8)\end{array}$ & $\begin{array}{c}2,7 \\
(2,2 ; 3,2)\end{array}$ & $\begin{array}{c}2,54 \\
(2,0 ; 2,9)\end{array}$ \\
\hline Charme (0 ou 1$)$ & $\begin{array}{c}0,87 \\
(0,38 ; 1,38)\end{array}$ & $\begin{array}{c}0,87 \\
(0,35 ; 1,34)\end{array}$ & $\begin{array}{c}0,88 \\
(0,37 ; 1,36)\end{array}$ & $\begin{array}{c}0,85 \\
(0,34 ; 1,32)\end{array}$ \\
\hline \multicolumn{5}{|c|}{ Caractéristiques des arbres précédents les plus proches } \\
\hline $\begin{array}{l}\text { Qualité C + D } \\
(i-1)\end{array}$ & $\begin{array}{c}-1,0 \\
(-1,4 ;-0,7)\end{array}$ & & $\begin{array}{c}-1,0 \\
(-1,4 ;-0,7)\end{array}$ & \\
\hline \multicolumn{5}{|c|}{ Cumul des diamètres coupés sur $p$ précédents les plus proches } \\
\hline Cumul sur $p=3$ arbres & $\begin{array}{c}-0,0157 \\
(-1,1 ;-0,1)\end{array}$ & $\begin{array}{c}-0,0155 \\
(-1,1 ;-0,1)\end{array}$ & & \\
\hline Log vraisemblance & $-120,39$ & $-122,84$ & $-123,4$ & $-126,0$ \\
\hline
\end{tabular}

Tableau II. Estimation (intervalle de confiance) des paramètres du modèle marginal.

\begin{tabular}{lcc}
\hline & $\begin{array}{c}\text { Modèle } \\
\text { retenu }\end{array}$ & $\begin{array}{c}\text { Modèle } \\
\text { caractéristiques } \\
\text { de l'arbre }\end{array}$ \\
\hline Constante & $-6,13$ & $-6,57$ \\
& $(-6,3 ;-5,8)$ & $(-6,8 ;-6,2)$ \\
Taux & $-22,5$ & $-18,4$ \\
& $(-30,6 ;-15,5)$ & $(-28,4 ;-9,4)$ \\
$20<$ diamètre $<40$ & 2,8 & 2,50 \\
& $(2,3 ; 3,1)$ & $(2,0 ; 2,9)$ \\
$40<$ diamètre $<60$ & 3,9 & 3,84 \\
& $(3,4 ; 4,3)$ & $(3,3 ; 4,3)$ \\
$60<$ diamètre & 4,8 & 4,96 \\
Qualité C & $(4,1 ; 5,4)$ & $(4,2 ; 5,7)$ \\
Qualité D & 1,7 & 2,1 \\
Charme $(0$ ou 1$)$ & $(1,3 ; 2,1)$ & $(1,6 ; 2,5)$ \\
Cumul des diamètres coupés sur $p$ précédents & 2,54 \\
Cumul sur $p=3$ arbres & $-0,0266$ & $(2,0 ; 2,9)$ \\
Log vraisemblance & 1,9 & 0,85 \\
\hline & $(1,5 ; 2,3)$ & $(0,34 ; 1,32)$ \\
& $0,044 ;-0,0011)$ & $-126,0$ \\
& $-121,4$ &
\end{tabular}

Le coefficient du cumul des diamètres est significatif avec un $\mathrm{khi}^{2}$ à 1 d.d.l de $2 \times(126,0-120,4)=11,2$ soit une $p$ value inférieure à $5^{0 / 00}$.

On peut observer que les valeurs des coefficients sont qualitativement peu différentes de celles du modèle (1).

\section{2. Étude de la variabilité de la probabilité de coupe (modèle conditionnel)}

\subsubsection{Description de l'influence des décisions précédentes ( $Y_{i}^{-}$connu)}

Par simulation, nous avons calculé les probabilités de coupe individuelle $P_{i}$ (cf. ci-dessus 2.4.1). Les différences $P_{i}-C_{i}$ sont présentées figure $1 \mathrm{a}$, en fonction de la distance parcourue depuis le début du martelage.

Elles valent généralement quelques pourcents. Les plus fortes valeurs négatives correspondent à des arbres dont les précédents ont été conservés, bien qu'ils aient une forte probabilité de coupe. Inversement, les plus fortes différences positives correspondent à des arbres dont les précédents ont été coupés mais avaient une forte probabilité d'être conservés.

On remarque que les différences proche de zéro se trouvent un peu plus concentrées en milieu de parcours. Elles correspondent à des séries d'arbres de faible dimension et à fort taux de rentabilité, ayant une forte probabilité de conservation et qui ont été conservé.

La figure $1 \mathrm{~b}$ présente les différences $P_{i}-C_{i}$ pour le sousensemble des arbres martelés par l'opérateur. Pour la majorité, l'écart est négatif : ces arbres ont été martelé pour leurs 


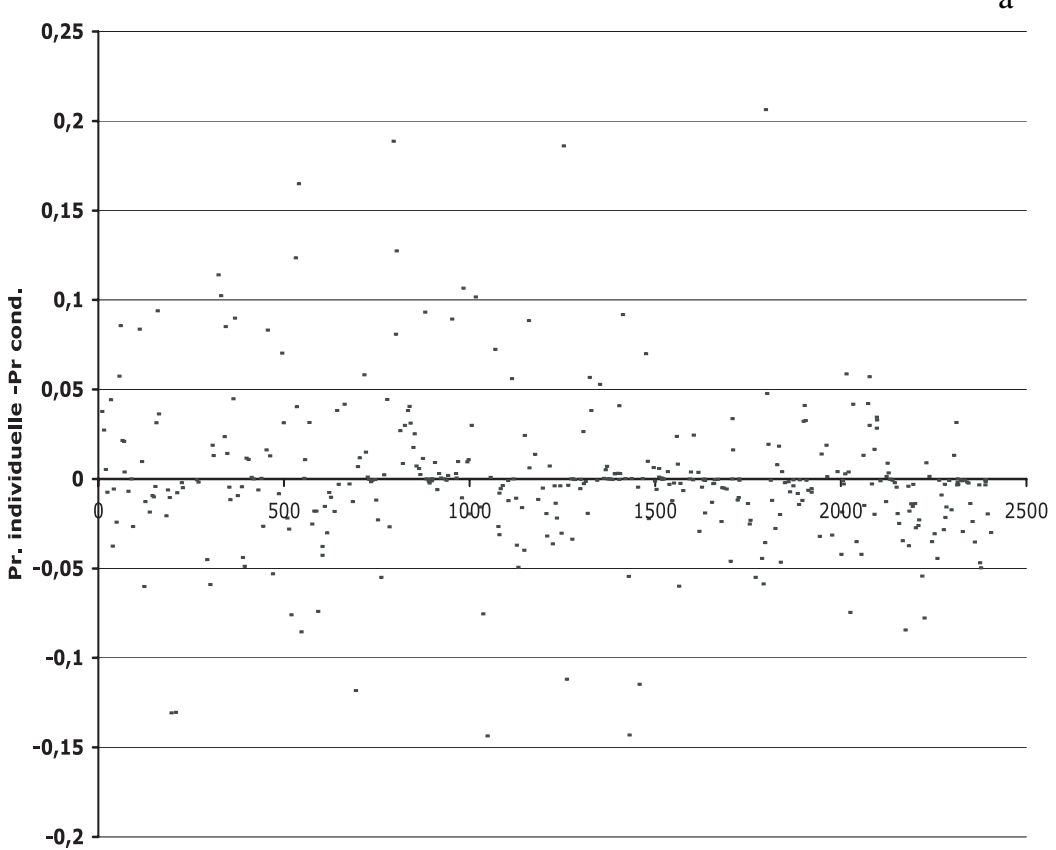

Distance parcourue

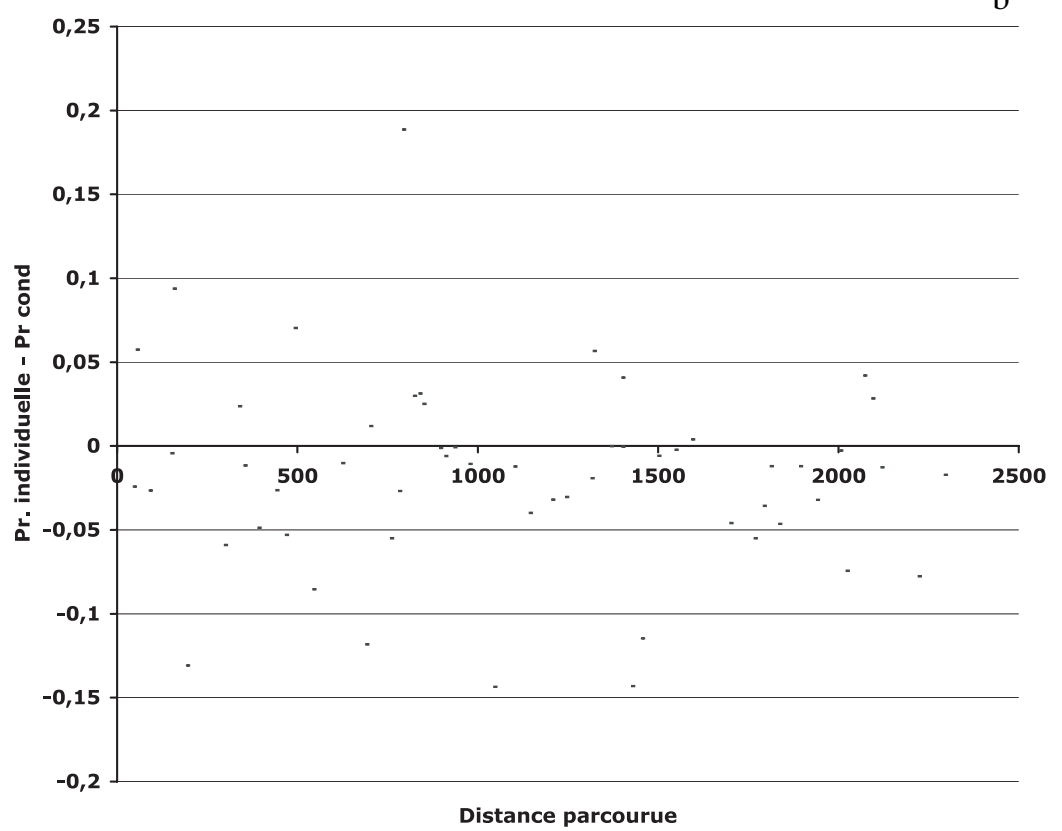

Figure 1. Différence Probabilité individuelle - Probabilité conditionnelle en fonction de la distance parcourue depuis le début du martelage. (a) Ensemble des arbres (b) arbres martelés. caractéristiques individuelles, mais aussi du fait que les arbres précédents ont été conservés. Néanmoins, pour 13 arbres (sur 58) l'écart est positif ; la coupe de l'un des deux arbres précédents n'a pas empêché le martelage (parmi eux, il y a les cas d'arbres « jumeaux » tous les deux martelés).

\subsubsection{Description a priori (décisions $Y_{i}^{-}$inconnues)}

Pour chaque arbre, nous présentons figure 2 la variance des probabilités conditionnelles $\operatorname{Var}(i)=\Sigma\left(C_{i}-P_{i}\right)^{2} \times \mathrm{P}\left(Y_{i}^{-}\right)$, divisée par la variance $P_{i} \times\left(1-P_{i}\right)$.
Ces pourcentages sont généralement assez faibles, essentiellement parce que la décision de conservation est prise très souvent et donc il n'y a que peu de possibilités de modulation. Néanmoins, cette figure permet de repérer les arbres où les décisions sont susceptibles de varier avec les décisions précédentes.

\subsubsection{Variation selon le cheminement dans la parcelle}

Nous avons recalculé les valeurs de $P_{i}$ en changeant le cheminement de l'opérateur dans la parcelle. La variation de $P_{i}$ entre deux cheminements est présentée en fonction de la distance 


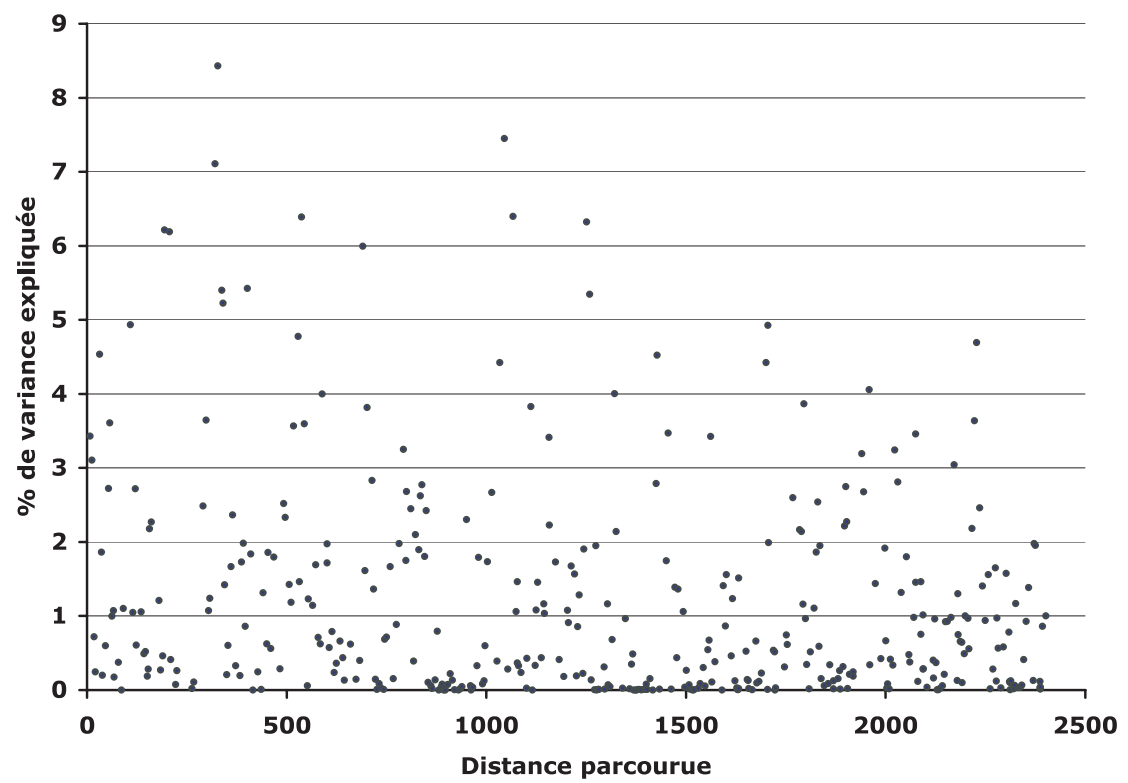

Figure 2. Pour chaque arbre, $\%$ de variance expliquée par les décisions précédentes.

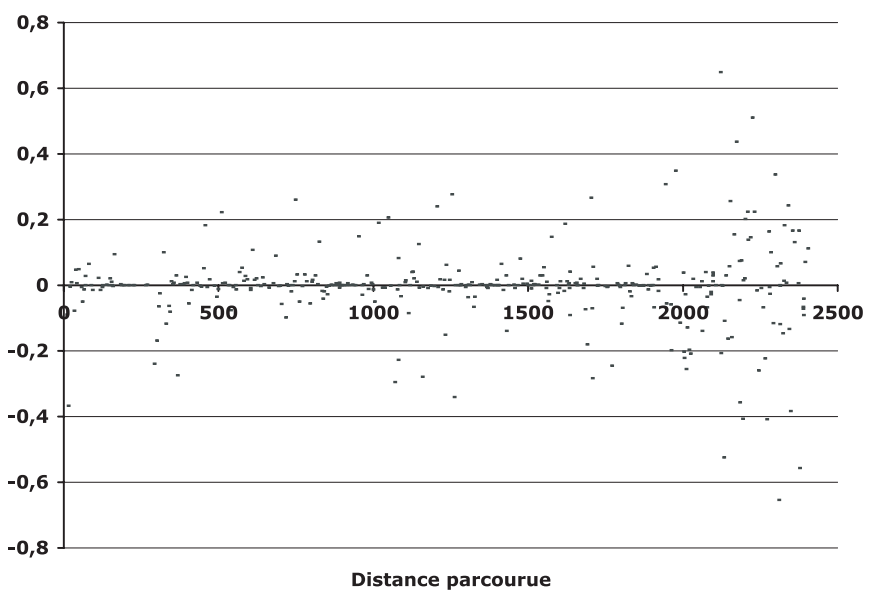

Figure 3. Variation des probabilités individuelles entre deux cheminements.

parcourue (Fig. 3). On constate que cette variation est relativement faible pour beaucoup d'arbres et que les arbres présentant une forte variation sont plutôt situés en fin de parcours. Sur le terrain, cette zone semble un peu plus hétérogène, ce qui peut expliquer que l'influence du parcours y soit plus importante.

\subsubsection{Variation selon le paramètre c}

Pour chaque arbre, la figure 4 présente $\operatorname{Var}(i)$ pour 2 valeurs du paramètre $c$. Comme attendu, $\operatorname{Var} C_{i}$ croît avec ce paramètre.

\section{3. Étude de la récolte par simulation de martelage}

\subsubsection{Simulation de comportements de l'opérateur}

Le modèle (1) a été simulé avec les paramètres du modèle présenté tableau Ib de façon à estimer la variabilité de la coupe.

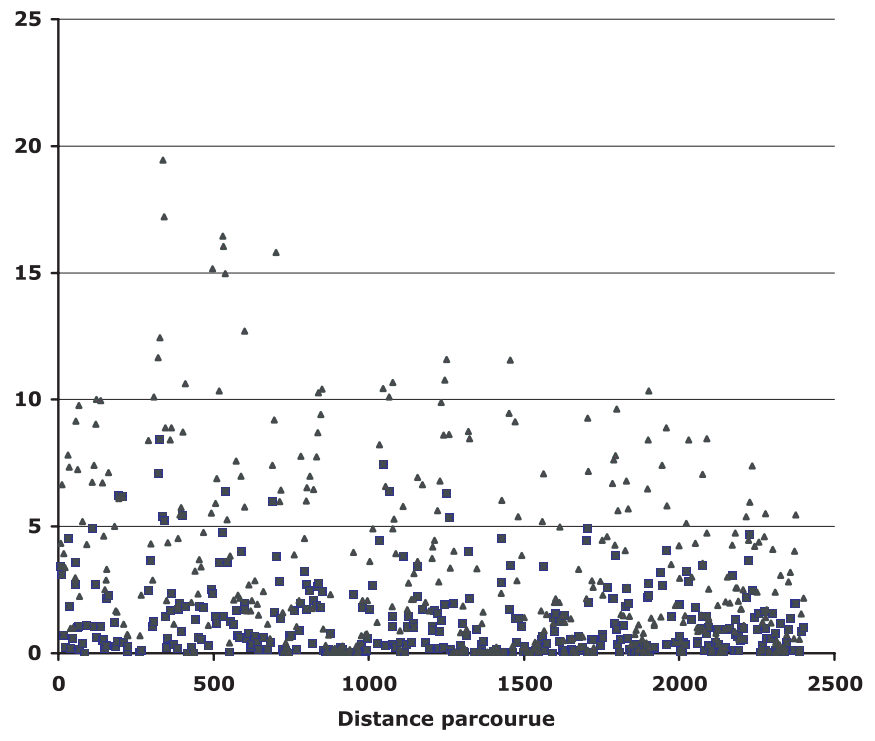

Figure 4. Pour chaque arbre, variance des probabilités conditionnelles pour 2 valeurs du parametre c.

Le tableau III montre les effectifs coupés simulés par catégorie de diamètre, par catégorie de qualité et de diamètre $X$ qualité. On observe des écarts raisonnables entre les effectifs simulés et ceux coupés par l'opérateur. Les écarts-types sont relativement grands, de peu inférieurs à ceux correspondant au modèle binomial.

Avec un autre parcours, les écarts sont plus importants. Cette variabilité conduit à penser que la comparaison de deux opérateurs sera délicate si l'on compare leurs récoltes et qu'il sera préférable de comparer leurs paramètres.

Lorsque les paramètres $c$ augmentent, une plus grande agrégation spatiale des coupes et une augmentation générale de la probabilité de coupe sont attendues : ce point est précisé par la 
Tableau III. Effet du martelage : Effectif avant et après coupe.

\begin{tabular}{|c|c|c|c|c|c|}
\hline & $\begin{array}{l}\text { Parcelle avant } \\
\text { coupe }\end{array}$ & $\begin{array}{l}\text { Coupé par } \\
\text { l'opérateur }\end{array}$ & $\begin{array}{l}\text { Simulation } \\
\text { modèle (1) } \\
\text { (écart-type) }\end{array}$ & $\begin{array}{l}\text { Simulation } \\
\text { modèle (1) } \\
2^{\mathrm{e}} \text { parcours } \\
\text { (écart-type) }\end{array}$ & $\begin{array}{l}\text { Simulation } \\
\text { modèle marginal } \\
\text { (écart-type) }\end{array}$ \\
\hline \multicolumn{6}{|c|}{ Effectif par catégorie de diamètre } \\
\hline Diamètre $<20$ & 96 & 1 & $1,4(1)$ & $1,2(1,1)$ & $0,8(0,8)$ \\
\hline $20<$ diamètre $<40$ & 173 & 21 & $21,8(4,1)$ & $20,4(3,9)$ & $22,0(4,0)$ \\
\hline $40<$ diamètre $<60$ & 91 & 22 & $21,8(3,9)$ & $23,8(3,5)$ & $21,9(3,3)$ \\
\hline $60<$ diamètre & 40 & 14 & $13,4(3,4)$ & $14,8(2,7)$ & $13,5(2,5)$ \\
\hline \multicolumn{6}{|c|}{ Effectif par catégorie de qualité } \\
\hline Qualité A + B & 60 & 3 & $3,7(1,7)$ & $3,8(1,7)$ & $3,9(1,8)$ \\
\hline Qualité C & 183 & 27 & $26,2(3,9)$ & $29,1(3,9)$ & $27,0(3,9)$ \\
\hline Qualité D & 157 & 28 & $28,5(4,0)$ & $27,2(4,0)$ & $27,1(3,9)$ \\
\hline \multicolumn{6}{|c|}{ Effectif par catégorie de diamètre $\times$ qualité } \\
\hline \multicolumn{6}{|l|}{ Diamètre $<20$} \\
\hline Qualité A + B & 7 & 0 & $0,0(0,1)$ & $0,0(0,1)$ & $0,0(0,1)$ \\
\hline Qualité C & 47 & 0 & $0,5(0,7)$ & $0,6(0,8)$ & $0,3(0,6)$ \\
\hline Qualité D & 42 & 1 & $0,8(0,9)$ & $0,5(0,7)$ & $0,4(0,6)$ \\
\hline \multicolumn{6}{|l|}{$20<$ diamètre $<40$} \\
\hline Qualité A + B & 11 & 0 & $0,1(0,2)$ & $0,1(0,3)$ & $0,3(0,3)$ \\
\hline Qualité C & 73 & 7 & $6,2(2,2)$ & $5,6(2,2)$ & $6,5(2,3)$ \\
\hline Qualité D & 89 & 14 & $15,5(3,4)$ & $14,7(3,3)$ & $15,4(3,3)$ \\
\hline \multicolumn{6}{|l|}{$40<$ diamètre $<60$} \\
\hline Qualité A + B & 23 & 0 & $0,9(0,9)$ & $1,0(1)$, & $1,1(1,0)$ \\
\hline Qualité C & 45 & 12 & $10,9(2,6)$ & $12,9(2,8)$ & $11,3(2,6)$ \\
\hline Qualité D & 23 & 10 & $10,0(2,2)$ & $9,80(2,2)$ & $9,4(2,3)$ \\
\hline \multicolumn{6}{|l|}{$60<$ diamètre } \\
\hline Qualité A + B & 19 & 3 & $2,7(1,4)$ & $2,6(1,4)$ & $2,8(1,5)$ \\
\hline Qualité C & 18 & 8 & $8,5(1,9)$ & $9,9(2,1)$ & $8,8(2,0)$ \\
\hline Qualité D & 3 & 3 & $2,1(0,7)$ & $2,1(1,0)$ & $1,8(0,9)$ \\
\hline
\end{tabular}

figure 5 qui présente les effectifs coupés en fonction du paramètre $c$.

\subsubsection{Simulation à objectifs fixés}

Le modèle marginal permet de simuler une stratégie répondant à des objectifs globaux fixés au niveau de la parcelle. Les résultats sont présentés tableau IV. En général, les objectifs de coupe sont réalisés. Les écarts-types sont relativement grands, de peu inférieurs à ceux correspondant au modèle binomial. Si l'opérateur prend davantage en compte les décisions précédentes, les écarts-types diminuent relativement peu.

Lorsque les paramètres $g$ augmentent, une plus grande agrégation spatiale des coupes est attendue : il y a augmentation de $P e$ la probabilité de coupe sachant que les arbres précédents sont enlevés. Toutefois, il y a diminution de $P c$, la probabilité de coupe sachant que les arbres précédents sont conservés, $P c$ et $P e$ étant liées par la contrainte « $M_{i}$ moyenne des $T_{i} »$. Le niveau de coupe reste constant, mais la variabilité des probabilités de coupe devient plus importante.

Lorsque $g$ devient très grand, la fluctuation est limitée soit que $P c$ atteint 1 soit que $P e$ atteint 0 . Deux types d'arbres peuvent alors être distingués : dans le premier type, $P c=1$, l'arbre

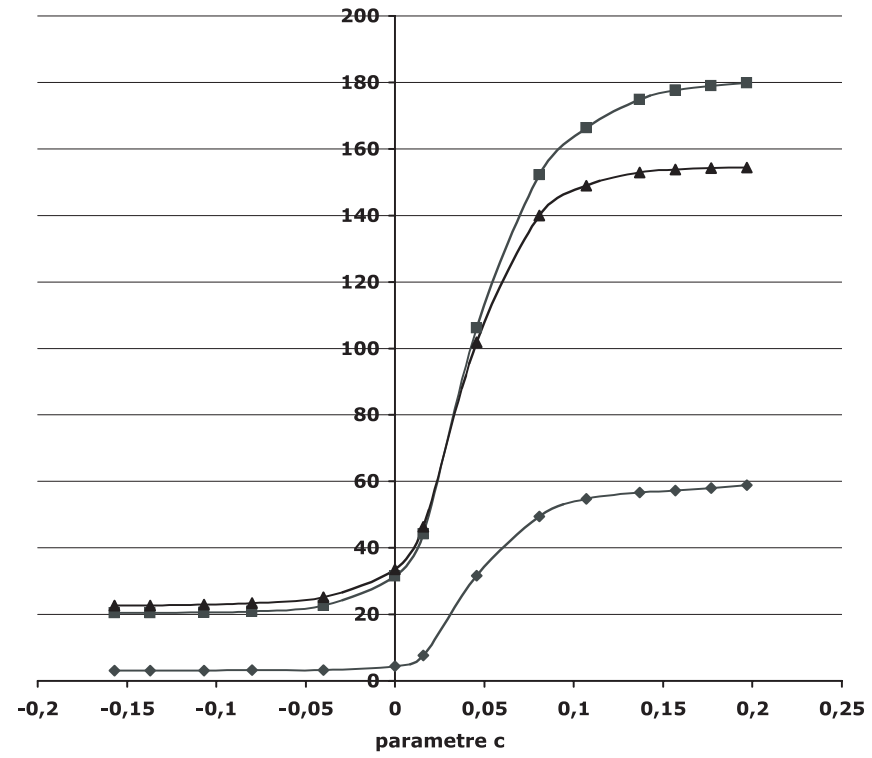

Figure 5. Effectifs coupés par qualité en fonction du paramètre de dépendance des décisions (modèle conditionnel). 
Tableau IV. Simulation de martelage à objectif déterminé (modèle marginal).

\begin{tabular}{|c|c|c|c|c|c|}
\hline & $\begin{array}{c}\text { Proportion de coupe } \\
\text { souhaitée } \\
\text { (logit) }\end{array}$ & $\begin{array}{c}\text { Effectif coupé } \\
\text { souhaité }\end{array}$ & $\begin{array}{c}\text { Effectif coupé simulé } \\
\text { pour } g=-2 \\
\text { (écart-type) }\end{array}$ & $\begin{array}{c}\text { Effectif coupé simulé } \\
\text { pour } g=0 \\
\text { (écart-type) }\end{array}$ & $\begin{array}{c}\text { Effectif coupé simulé } \\
\text { pour } g=2 \\
\text { (écart-type) }\end{array}$ \\
\hline \multicolumn{6}{|c|}{ Effectif par catégorie de diamètre $\times$ qualité } \\
\hline \multicolumn{6}{|l|}{ Diamètre $<20$} \\
\hline Qualité A + B & $4 \%(-3,1780)$ & 0,28 & $0,27(0,5)$ & $0,28(0,5)$ & $0,27(0,5)$ \\
\hline Qualité C & $8 \%(-2,4423)$ & 3,8 & $3,8(1,8)$ & $3,7(1,9)$ & $3,7(5)$ \\
\hline Qualité D & $10 \%(-2,1972)$ & 4,2 & $4,2(1,9)$ & $4,2(1,9)$ & $4,2(5)$ \\
\hline \multicolumn{6}{|l|}{$20<$ diamètre $<40$} \\
\hline Qualité A + B & $10 \%(-2,1972)$ & 1,1 & $1,1(0,9)$ & $1,1(1,0)$ & $1,1(1)$ \\
\hline Qualité C & $15 \%(-1,7346)$ & 10,9 & $10,9(2,8)$ & $10,9(3,0)$ & $10,8(10)$ \\
\hline Qualité D & $19 \%(-1,4500)$ & 16,9 & $17,0(3,3)$ & $16,9(3,7)$ & $16,7(14)$ \\
\hline \multicolumn{6}{|l|}{$40<$ diamètre $<60$} \\
\hline Qualité A + B & $15 \%(-1,7346)$ & 3,45 & $3,46(1,7)$ & $3,4(1,7)$ & $3,4(3)$ \\
\hline Qualité C & $22 \%(-1,2657)$ & 9,9 & $9,9(2,4)$ & $9,9(2,8)$ & $9,7(7)$ \\
\hline Qualité D & $27 \%(-0,9946)$ & 6,2 & $6,2(2,1)$ & $6,2(2,1)$ & $6,2(4)$ \\
\hline \multicolumn{6}{|l|}{$60<$ diamètre } \\
\hline Qualité A + B & $20 \%(-1,3863)$ & 3,8 & $3,8(1,7)$ & $3,8(1,7)$ & $3,8(3)$ \\
\hline Qualité C & $29 \%(-0,8954)$ & 5,2 & $5,2(1,9)$ & $5,2(1,9)$ & $5,2(3)$ \\
\hline Qualité D & $34 \%(-0,6633)$ & 1,0 & $1,0(0,8)$ & $1,0(0,8)$ & $1,0(0,8)$ \\
\hline \multicolumn{6}{|c|}{ Effectif par catégorie de diamètre } \\
\hline Diamètre $<20$ & $8,6 \%$ & 8,3 & $8,3(2,5)$ & $8,2(2,7)$ & $8,2(9)$ \\
\hline $20<$ diamètre $<40$ & $16,7 \%$ & 28,9 & $28,9(3,9)$ & $28,9(4,9)$ & $28,6(24)$ \\
\hline $40<$ diamètre $<60$ & $21,5 \%$ & 19,6 & $19,5(3,1)$ & $19,6(3,9)$ & $19,4(13)$ \\
\hline $60<$ diamètre & $25 \%$ & 10 , & $10,0(2,5)$ & $10,0(2,7)$ & $10,0(6)$ \\
\hline \multicolumn{6}{|c|}{ Effectif par catégorie de qualité } \\
\hline Qualité A + B & $14,3 \%$ & 8,6 & $8,56(2,6)$ & $8,6(2,6)$ & $8,5(8)$ \\
\hline Qualité C & $16,6 \%$ & 30,0 & $29,8(4,0)$ & $29,8(4,9)$ & $29,4(23)$ \\
\hline Qualité D & $18,4 \%$ & 28,3 & $28,3(3,9)$ & $28,4(4,7)$ & $28,1(22)$ \\
\hline
\end{tabular}

est toujours coupé si les précédents sont conservés et est susceptible d'être coupé lorsque les précédents sont coupés (Éq. (3)). Dans le second type, $P e=0$, l'arbre n'est coupé que si ces précédents ne le sont pas. Les effectifs de chaque population dépendent des probabilités marginales et donc du taux de coupe défini par l'opérateur (résultats non présentés).

\section{CONCLUSIONS ET PERSPECTIVES}

Cet article présente deux modèles permettant d'expliquer, soit le comportement local d'un opérateur, soit la récolte moyenne sur la parcelle.

Le modèle conditionnel permet de tester différentes hypothèses relatives au comportement d'un opérateur, notamment celles relatives à la prise en compte du voisinage et des décisions précédentes. Par simulation, les conséquences d'une stratégie de coupe peuvent être examinées sur les moyenne et structure du peuplement. Toutefois, les paramètres précisant la dépendance entre décisions font varier les probabilités de coupe.

Avec un modèle marginal, il est possible de tester des facteurs expliquant la récolte ou de simuler un martelage visant une récolte donnée avec différentes dépendances entre décisions (paramètres $g$ ) ou différents parcours. Ce modèle offre une réelle souplesse du fait que les liaisons entre décisions peuvent être choisies indépendamment des autres paramètres.

Pour prolonger ces recherches, plusieurs directions sont envisageables.

D'une part, il est envisagé de comparer des opérateurs entre eux ou par rapport à des comportements optimisant un ou plusieurs critères (financiers, environnementaux ou normes forestières), en imposant le cheminement ou en laissant celui-ci au choix du sylviculteur. Ensuite, les conditions expérimentales pourraient varier de façon à comparer les stratégies de martelage dans des peuplements de structures différentes.

D'autre part, d'autres variables comme des indices de compétition, pourraient être introduites dans le modèle pour l'adapter à d'autres contextes sylvicoles.

Enfin, lorsque le parcours suivi par l'opérateur n'a pas été enregistré, un modèle purement spatial de répartition des arbres coupés est envisageable.

Dans toutes ces perspectives, le marteloscope apparaît comme un moyen précieux pour planifier des « expériences » 
permettant d'étudier spécifiquement le comportement du sylviculteur sur le terrain.

Remerciements : Nous remercions A. Ducret d'avoir réalisé le programme de calcul nécessaire à cet article. Nous remercions également les deux lecteurs qui ont examiné le manuscrit et apporté leurs commentaires constructifs.

\section{ANNEXE}

Calcul de la vraisemblance $\mathrm{V}$ pour les paramètres connus.

$$
\begin{gathered}
V=P\left(Y_{1}=y_{1}, \ldots Y_{I}=y_{I} / X\right) \\
V=P\left(Y_{I}=y_{I} / Y_{I-1}=y_{I-1}, \ldots Y_{1}=y, X\right) \times P\left(Y_{1}=y_{1}, \ldots Y_{I-1}=y_{1-1} / X\right) .
\end{gathered}
$$

Avec les propriétés markoviennes on a :

$V=P\left(Y_{I}=y_{i} / Y_{I-1}=y_{I-1}, y_{1-2}=y_{1-p}, X_{I}\right) \times P\left(Y_{1}=y_{1}, \ldots Y_{I-1}=y_{1-1} / X\right)$.

En recommençant on obtient :

$$
\begin{gathered}
P\left(Y_{p}=y_{p}, \ldots Y_{1}=y_{1} / X\right) \prod_{i=p+1}^{I} P\left(Y_{i}=y_{i} / Y_{i-1}=y_{i-1}, y_{i-2}, X_{i}\right) \\
P\left(Y_{p}=y_{p}, \ldots Y_{1}=y_{1} / X\right) \prod_{i=p+1}^{I} P\left(Y_{i}=y_{i} / Y_{i-1}=y_{i-1}, Y_{i-2}=y_{i-2}\right) \\
P\left(Y_{p}=y_{p}, \ldots Y_{1}=y_{1} / X\right) \prod_{i=p+1}^{l}\left(C_{i}\right)^{y_{i}}\left(1-C_{i}\right)^{1-y_{i}} .
\end{gathered}
$$

Soit encore

$$
\begin{aligned}
\log (V)= & \log \left(P\left(Y_{p}=y_{p}, \ldots Y_{1}=y_{1} / X\right)\right) \\
& +\sum_{i=p+1}^{I} y_{i} \operatorname{Logit} C_{i}-\log \left(1+\exp \left(\operatorname{Logit} C_{i}\right)\right) .
\end{aligned}
$$

Nous supposerons que les $p$ premiers arbres sont conservés de façon certaine $\left(P\left(P_{p+1}^{-}=0\right)=P\left(Y_{p}=0, \ldots, Y_{1}=0\right)=1\right)$.

Cette supposition permet d'une part de calculer $\log \left(P\left(Y_{p}=y_{p}, \ldots Y_{1}=y_{1}\right)\right)$ et d'autre part d'initialiser le calcul qui suit. Par ailleurs, nous avons vérifié que cette supposition n'a pas d'influence sur l'optimisation.

Pour terminer le calcul, nous devons connaître $\operatorname{Logit}\left(C_{i}\right)$. Nous utiliserons la formule (2), les termes $d_{i}$ étant calculés les uns après les autres, en effectuant les deux étapes suivantes.

- Résolution de l'équation implicite que doit satisfaire $d_{i}$ :

La moyenne de $C_{i}$ sur la distribution de $Y_{i}^{-}$doit être égale à la valeur Mi donnée par (1). $d_{i}$ est donc la solution de l'équation :

$$
M_{i}=\sum_{w_{1} \ldots w_{p}} P\left(Y_{i}=1 / \overline{Y_{i}}=w_{1}, \ldots w_{p}\right) P\left(\overline{Y_{i}}=w_{1}, \ldots w_{p}\right) ;
$$

les $w_{1}, \ldots, w_{p}$ parcourant les $2^{\mathrm{p}}$ états possibles.

Les paramètres $\alpha$ étant, la valeur du membre de gauche est connue.
Dans le membre de droite, le premier terme sous le signe de sommation est donné par (2). Il contient l'inconnue $d_{i}$. Le second terme (la loi de probabilité de $Y_{i}^{-}$) est connu initialement ou sera donné par l'étape 2 de l'itération précédente.

L'inversion de cette équation pose un problème numérique pour les probabilités très proche de 1 ou de 0 ; pour le résoudre, nous avons posé $\exp (z) /(1+\exp (z))=1$ pour $z>20$.

- Mise à jour de la loi de probabilité de $Y_{i}^{-}$:

Pour chaque $w_{1}, \ldots, w p$ possible on a :

$$
\begin{aligned}
P\left(\overline{Y_{i+1}^{-}}=w_{1}, \ldots w_{p}\right) & =\sum_{k=0}^{1} P\left(Y_{i+1}^{-}=w_{1}, \ldots w_{p} \text { et } Y_{i-p-1}=k\right) \\
P\left(Y_{i+1}^{-}=w_{1}, \ldots w_{p}\right) & =\sum_{k=0}^{1} P\left(Y_{i}=w_{1} / Y_{i}^{-}\right. \\
& \left.=w_{2}, \ldots w_{p}, k\right) P\left(Y_{i}^{-}=w_{2}, \ldots w_{p}, k\right) .
\end{aligned}
$$

Le premier terme sous le signe de sommation est donné par (2) $\left(d_{i}\right.$ vient d'être calculé) ; le second terme est connu initialement ou est donné par l'itération précédente.

Remarques :

Il faut noter que le calcul de $d_{i}$ ne fait pas intervenir les valeurs de $Y$ observées, dès lors que les paramètres $\alpha$ et $g$ sont connus.

D'autre part, comme le souligne Heagerty (2002), la complexité des calculs croît de façon exponentielle avec l'ordre $\mathrm{p}$, en $\mathrm{I} \times 2^{\mathrm{p}}$. Avec un ordinateur de gamme moyenne, pour $p=8$ et $n=400$ le temps de calcul est de quelques minutes.

\section{RÉFÉRENCES}

[1] Baylot J., Vautherin P., Classement des bois ronds feuillus, Publication du CTBA, Paris, 1992.

[2] Delacre F., Corroy M. Un martellodrome en forêt domaniale de Florennes, Cahier Technique $\mathrm{N}^{\circ} 19, \mathrm{~N}^{\circ} 57$, Mars/Avril, Forêt Wallonne, 2002.

[3] Diggle P.J., Heagerty P.J., Liang K., Zeger S.L., Analysis of Longitudinal Data, Oxford University Press, 2003.

[4] Heagerty P.J., Marginalized transition models and likelihood inference for longitudinal categorical data, Biometrics 58 (2002) 342$351,2002$.

[5] Nelder J.A., Mead R., A simplex method for function Minimization, Comput. J. 7 (1964) 308-313.

[6] Pierrat J.C., Structuration d'un peuplement hétérogène au moyen d'éclaircies locales Ann. For. Sci. (2004) 179-190.

[7] Sédillot C., Tomasini J., Analyse des données d'un marteloscope en futaie irrégulière résineuse (ONF Alsace), Document interne du LERFOB, 2000.

[8] Zucchini W., Gadow K.v., Two indices of agreement among foresters selecting trees for thinning, For. Landsc. Res. 1 (1995) 199-206. 